\title{
Nursing home-acquired pneumonia: a 10 year single-centre experience
}

\author{
E Polverino, ${ }^{1}$ P Dambrava, ${ }^{1}$ C Cillóniz, ${ }^{1}$ V Balasso, ${ }^{2}$ M A Marcos, ${ }^{3}$ C Esquinas, ${ }^{1}$ \\ J Mensa, ${ }^{2}$ S Ewig, ${ }^{4}$ A Torres ${ }^{1}$
}

${ }^{1}$ Respiratory Department, Hospital Clinic-IDIBAPS, Barcelona, Spain

${ }^{2}$ Servei de Malalties Infeccioses, Hospital Clínic, Barcelona, Spain

${ }^{3}$ Servei de Microbiología, Hospital Clínic, Barcelona, Spain

${ }^{4}$ Thoraxzentrum Ruhrgebiet Kliniken für Pneumologie und Infektiologie Ev. Krankenhaus Herne und

Augusta-Kranken-Anstalt, Bochum, Germany

\section{Correspondence to}

Professor Antoni Torres, Institut Clínic de Pneumologia i Cirurgia Toràcica, Hospital Clinic, Villarroel 170, E-8036 Barcelona, Spain; atorres@clinic.ub.es

Received 3 August 2009 Accepted 28 January 2010
Background Pneumonia among nursing home (NH) residents has increased considerably in recent years, but it remains unclear whether it should be considered as community-acquired pneumonia (CAP) or a new category of infection.

Methods 150 consecutive cases of $\mathrm{NH}$-acquired pneumonia (NHAP) (from 1 February 1997 to 1 July 2007) were analysed.

Results Patients (median age, 82 years; range, 77-87 years) showed numerous co-morbidities, (neurological, 55\%; pulmonary, 38\%; cardiac, 35\%) and severe disability for daily activities (partial, $32 \%$; total, $31 \%)$. Cases of NHAP were mainly classified as mild to moderate according to the CRB-65 score (CRB-65 classes 0-1 and 2, 41\% each). In-hospital and 30-day mortality were $8.7 \%$ and $20 \%$, respectively. Aetiology was defined in 57 cases (38\%). The most common isolates were Streptococcus pneumoniae (58\%), Enterobacteriaceae (Gram-negative bacteria (GNB)) (9\%), atypical bacteria (7\%), respiratory viruses (5\%), methicillin-resistant Staphylococcus aureus (MRSA) (5\%) and Legionella pneumophila (5\%). The most frequent causes of treatment inadequacy were use of $\beta$-lactams alone (25\%) and lack of aspiration assessment (15\%). Prognostic factors of 1-month mortality were neurological comorbidities (OR 4.5; 95\% Cl 1.3 to 15.7; $p=0.020$ ), septic shock (OR 6.6; $95 \% \mathrm{Cl} 1.3$ to 34.0 ; $p=0.025$ ), pleural effusion (OR 3.6; $95 \% \mathrm{Cl} 1.1$ to 11.7 ; $\mathrm{p}=0.036$ ) and isolation of GNB or MRSA (OR 16.4; $95 \%$ Cl 2.1 to 128.9; $p=0.008$ ).

Conclusions The patients show clinical characteristics (eg, age and co-morbidities) comparable with those with hospital-acquired pneumonia. However, microbiological and mortality data of patients with NHAP are more similar to the data of those with CAP. Isolation of GNB or MRSA was associated with increased mortality risk. CAP empirical antibiotic coverage is still indicated in NHAP, although specific risk factors for multidrug-resistant infections should be assessed on an individual basis.

\section{INTRODUCTION}

Nursing home-acquired pneumonia (NHAP) is probably the largest subgroup of healthcare-associated pneumonia (HCAP), and the number of cases has increased in recent decades, with the worldwide diffusion of long-term care facilities (LTCFs). ${ }^{1}$ Moreover, the number of older individuals living in nursing homes (NHs) is expected to increase dramatically in the next 30 years, as $40 \%$ of adults will probably reside in an LTCF in later life. ${ }^{2}$ Pneumonia is the second most common infection in $\mathrm{NH}$ and the leading cause of mortality and hospitalisation. $^{3-7}$ Much information has been gathered on NHAP since the $1970 s^{6-8}$ : patients with NHAP are usually elderly, with multiple diseases (eg, cardiovascular, respiratory and neurological) and poor functional status. ${ }^{9-11}$ The clinical presentation of NHAP is often unusual, 6791213 with frequent extrapulmonary manifestations (mental confusion and gastrointestinal disorders), ${ }^{14}$ and the clinical presentation may be worse than in community-acquired pneumonia (CAP) (eg, hypoxaemia and altered consciousness). ${ }^{11} 1516$ The mortality rate of NHAP is close to that of hospitalacquired pneumonia (HAP) $)^{7}{ }^{11} 1^{15-17}$ (20-40\%), while its annual incidence is 30-fold that of the general population and 11-fold that for the elderly ( $\geq 75$ years). ${ }^{4} 71819$

A major concern has emerged regarding the microbial aetiology of NHAP, since two large retrospective studies on HCAP have reported an elevated incidence of pathogens common in nosocomial infections (Gram negative bacilli (GNB), or methicillin-resistant Staphylococcus aureus (MRSA) ${ }^{1020}$ and, particularly, of multidrug-resistant (MDR) microorganisms, ${ }^{21} 22$ which may justify nosocomial antibiotic coverage on admission. $^{1}$

In contrast, two prospective European studies described Streptococcus pneumoniae and Haemophilus influenzae as the most frequent pathogens in NHAP. $^{9} 1117$

Therefore, the microbial aetiology of NHAP is still under debate, while the effect of comorbidities, functional status, abnormal clinical presentation and microbial aetiology on the high mortality of HCAP is unclear.

The first objective of our study was to investigate microbial aetiology in NHAP and, particularly, the frequency of MDR microorganisms. Hence, the adequacy of empirical antibiotic therapy in our hospital was also investigated in order to review the current antibiotic treatment recommendations.

Secondly, we analysed possible risk factors for MDR infections, such as demographic, clinical and biochemical data, and severity scores.

Lastly, we investigated possible prognostic factors of mortality, including demographic, clinical and biochemical data on admission, and microbial aetiology.

\section{MATERIALS AND METHODS Study population}

We prospectively studied all consecutive cases of NHAP admitted to Hospital Clinic, Barcelona, Spain, from 1 February 1997 to 1 July 2007. 
In Spain, $\mathrm{NH}$ are currently considered institutions dedicated to assist individuals unable to perform routine daily activities autonomously, including toileting, eating and mobility. $\mathrm{NH}$ assistance usually includes part-time medical and physiotherapy support and full-time nursing care.

The diagnosis of pneumonia was made by the Emergency department (ED) doctor and confirmed by the respiratory physician in charge of recruitment and data collection for the study. The choice of empirical antibiotic treatment was taken exclusively by the attending physician. Immunosuppressed patients (neoplasia, severe haematological disorders, HIV, immunosuppressant treatment, chemotherapy in the last year) were excluded.

\section{Data collection}

Demographic data, comorbid illness (eg, chronic cardiovascular diseases, diabetes mellitus, chronic liver disease, renal failure, dementia and neurological diseases), previous antibiotic treatment and relevant data from the clinical history (eg, use of inhaled/systemic corticosteroids, influenza and pneumococcal vaccinations, smoking, alcohol intake, previous pneumonia, aspiration evidence and antibiotic allergies) were recorded in an Access database. Neurological disorders include degenerative diseases, multiple sclerosis, amyotrophic lateral sclerosis, Parkinson disease, Down syndrome, stroke and postanoxia brain injury. Autonomy for routine daily activities, including toileting, eating and mobility, was classified as follows: total (no need for external help), partial (need for partial help) and none (totally dependent on external help).

The following parameters were also recorded on admission: days of clinical course before admission, time elapsed in $\mathrm{ED}$, clinical symptoms, vital signs, laboratory data, chest x-ray (number of affected lobes, infiltrate radiographic pattern and localisation, presence of pleural effusion/atelectasis/cavitations), and PSI (Pneumonia Severity Index) and CRB-65 (confusion, respiratory rate, low blood pressure, age $\geq 65$ years) prognostic scales.

Relevant data on clinical course were recorded, including treatment (antibiotics and systemic corticosteroids, time of first doses from admission, compliance with the American guidelines for CAP management ${ }^{23}$ ), length of hospital stay, oxygen (arterial oxygen pressure $\left(\mathrm{PaO}_{2}\right)$ / fractional inspired oxygen $\left(\mathrm{FiO}_{2}\right)$ ) and ventilatory support (mechanical ventilation, non-invasive ventilation), day of clinical stability according to American Thoracic Society (ATS)/Infectious Diseases Society of America (IDSA) criteria, ${ }^{23}$ pulmonary (empyema, respiratory distress, pleural parapneumonic effusion, pneumothorax, surgical pleural draining) and extrapulmonary complications (cardiac arrhythmias, septic shock, acute renal failure, meningitis, endocarditis, SIADH (syndrome of inappropriate antidiuretic hormone secretion), positive/negative Clostridium diarrhoea, antibiotic secondary effects), in-hospital and 1-month mortality rates and cause of death, and treatment failure. ${ }^{23}$

We considered the treatment prescribed during the first $24 \mathrm{~h}$ of hospitalisation to be the initial treatment. An antibiotic regimen was defined as ATS adherent when the chosen antibiotics followed the recommendations included in the 2007 ATS guidelines, regardless of any additional antibiotic received. ${ }^{23}$

Appropriate first-line CAP antibiotic treatments were considered to be $\beta$-lactam+macrolide or a quinolone alone. Where aspiration was suspected, amoxicillin-clavulanic acid or piperacillintazobactam was considered appropriate antibiotic treatment.

\section{Microbiological data}

Within the first $24-48 \mathrm{~h}$ after admission, regular samples of sputum, blood for two cultures and serum for paired serology were taken (on admission and 4-6 weeks thereafter) for atypical pathogens (Chlamydia pneumoniae, Mycoplasma pneumoniae, Coxiella burnetii) and respiratory viruses. Tests for detection of urinary antigens for Legionella pneumophila and $S$ pneumoniae were systematically performed.

Respiratory secretion samples, including spontaneous sputum or bronchoalveolar lavage (BAL) fluid, fibreoptic bronchial aspirates (FBAS), tracheobronchial aspirates (TBAS) and pleural fluid, when available, were collected for Gram and ZiehlNielsen stains and for cultures for bacterial, fungal and mycobacterial pathogens.

\section{Sample processing}

Sample processing techniques are described in previous studies by our group. ${ }^{24}$

\section{Diagnostic criteria}

The aetiology of pneumonia was classified as presumptive if a valid sputum sample yielded $\geq 1$ predominant bacterial strains. Aetiology was considered definite if one of the following criteria was met: (1) blood cultures yielding a bacterial or fungal pathogen (in the absence of an apparent extrapulmonary focus); (2) pleural fluid cultures yielding a bacterial pathogen; (3) seroconversion (ie, a fourfold increase in immunoglobulin $G$ (IgG) titres) for $C$ pneumoniae and $L$ pneumophila $>1: 128$, C burnetii $>1: 80$, and respiratory viruses (ie, influenza viruses $A$ and $B$, parainfluenza viruses $1-3$, respiratory syncytial virus, adenovirus); (4) a positive urinary antigen for $L$ pneumophila or $S$ pneumoniae; and (5) bacterial growth in cultures of TBAS or FBAS $>10^{5} \mathrm{cfu} /$ $\mathrm{ml}$ and in BAL $>10^{4} \mathrm{cfu} / \mathrm{ml}$. A diagnosis of probable aspiration was made in cases of witnessed aspiration or in the presence of risk factors for aspiration (severely altered consciousness, abnormal swallowing).

We considered an uncommon microbial aetiology for CAP when GNB (ie, Escherichia coli, Klebsiella pneumoniae, etc), Pseudomonas aeruginosa or MRSA were isolated.

\section{STATISTICS}

Categorical variables were described using counts and percentages. Continuous variables were expressed as the mean $\pm \mathrm{SD}$, or median and IOR for abnormally distributed data (Kolmogorov-Smirnov test). Relationships between categorical variables were studied using the $\chi^{2}$ test, or Fisher exact test, when necessary. Comparison of continuous variables between two groups was carried out using the t test for unpaired data once normality was demonstrated; otherwise, the non-parametric test (Mann-Whitney $U$ test) was used. Univariate and multivariate logistic regression analyses were conducted to identify variables predictive of patients with potential first-line CAP antibiotic-resistant microorganisms (GNB or MRSA) (dependent variable). The independent variables were age, sex, length of stay, pneumonia in the previous year, suspected aspiration, inhaled corticosteroids, heart failure, chronic renal failure, diabetes mellitus, chronic liver disease, neurological disorders, chronic respiratory diseases, smoking, alcohol, autonomy for daily activities, pneumococcal vaccination, influenza vaccination, systemic corticosteroids, fever, dyspnoea, acute renal failure, shock, PCR, leucocytes $>12 \times 10^{9} / 1, \mathrm{PaO}_{2} / \mathrm{FiO}_{2}<200$, cavitation, atelectasis, pleural effusion, more than two affected lobes, PSI score and CRB-65 classes. Univariate and multivariate logistic regression analyses were performed to predict 30-day mortality (dependent variable). Independent variables were as above, including possible first-line CAP antibiotic-resistant 
microorganisms (GNB or MRSA). Variables that showed a significance in the univariate analysis $(p<0.1)$ were included in the multivariate logistic regression backward stepwise model to determine which of them were independently related to outcome. The Hosmer-Lemeshow goodness-of-fit test was performed to assess the overall fit of the model. All analyses were performed using SPSS 16 for Windows; a two-tailed $p$ value of $<0.05$ was considered statistically significant.

\section{RESULTS \\ Study population}

We analysed 150 consecutive cases of NHAP (median age 82 years (77-87 years); males, 49\%); Table 1 shows the main characteristics.

Median length of stay was 8 days (5-13 days). Only 20 patients $(13 \%)$ were admitted to the intensive care unit (ICU); their median length of stay was 11 days (9-23 days). Tables 2 and 3 show data on clinical presentation on admission (symptoms, analytical data and radiographic patterns) and severity indexes, respectively. NHAP were mainly classified as mild to moderate according to the CRB-65 score (CRB-65 classes $0-1$ and $2,41 \%$ each) but as moderate to severe by the Fine score (Fine classes 4 and 5, 33\% and 53\%) due to the weight of age in the PSI calculation.

In-hospital mortality was $8.7 \%(n=13)$ globally and $20 \%$ $(\mathrm{n}=30)$ after 1 month. Deceased patients had been mainly hospitalised in a ward ( $n=23 ; 77 \%)$; only five $(17 \%)$ were in an ICU. The most frequent respiratory complication was empyema $(n=5)$, followed by acute respiratory distress syndrome $(n=2)$ and pleural effusion $(n=2)$. Extrapulmonary complications included renal failure in nine cases (9\%), cardiac arrhythmia in six $(6 \%)$ and septic shock in five (5\%). Clinical stability was reached after a median of 6 days (4-9 days) of hospitalisation.

Aetiology was defined in $38 \%$ of cases $(n=57)$ (table 4$)$. The most common isolates were $S$ pneumoniae (58\%) and

Table 1 Main characteristics of the study population, $\mathrm{n}(\%)^{*}$

\begin{tabular}{|c|c|}
\hline \multicolumn{2}{|l|}{ Smoking habit } \\
\hline Non-smokers & $83(66)$ \\
\hline Smokers & $12(10)$ \\
\hline Ex-smokers & $31(25)$ \\
\hline \multicolumn{2}{|l|}{ Vaccinations } \\
\hline Pneumococcal vaccination & $15(15)$ \\
\hline Influenza vaccination & $75(75)$ \\
\hline \multicolumn{2}{|l|}{ Autonomy for daily activities } \\
\hline Full & $36(31)$ \\
\hline Partial & $38(32)$ \\
\hline Previous pneumonia in the last year & $23(18)$ \\
\hline Inhaled corticosteroids & 25 (19) \\
\hline Systemic corticosteroids & $4(3)$ \\
\hline \multicolumn{2}{|l|}{ Co-morbidities } \\
\hline Heart failure & $47(35)$ \\
\hline Chronic renal failure & $10(8)$ \\
\hline Diabetes mellitus & $28(21)$ \\
\hline Chronic liver disease & $3(2)$ \\
\hline Neurological disorders & $73(55)$ \\
\hline Chronic respiratory diseases & $51(38)$ \\
\hline \multicolumn{2}{|c|}{$\begin{array}{l}\text { Chronic respiratory diseases include: asthma (4\%), chronic bronchitis } \\
(9 \%) \text {, chronic obstructive pulmonary disease }(14 \%) \text {, ex tuberculosis } \\
\text { sequelae }(4 \%) \text {, pulmonary hypertension }(1 \%) \text { and others }(4 \%) \text {. } \\
\text { Neurological disorders include: degenerative diseases, multiple sclerosis, } \\
\text { amyotrophic lateral sclerosis, Parkinson disease, Down syndrome, } \\
\text { vascular cerebral accidents, postanoxia brain injury and dementia. } \\
{ }^{*} \text { Percentages of all cases with available information. }\end{array}$} \\
\hline
\end{tabular}

Table 2 Clinical presentation and laboratory data on admission

\begin{tabular}{|c|c|}
\hline \multicolumn{2}{|l|}{ Symptoms, n (\%)* } \\
\hline Previous 'common cold' symptoms & $49(38)$ \\
\hline Fever & $106(79)$ \\
\hline Chills & $46(36)$ \\
\hline Cough & $107(80)$ \\
\hline Purulent phlegm & $66(50)$ \\
\hline Pleuritic pain & $33(25)$ \\
\hline Dyspnoea & $102(76)$ \\
\hline Nausea/vomiting & $12(12)$ \\
\hline Altered mental status & $67(50)$ \\
\hline Acute renal failure & $22(17)$ \\
\hline Shock & $14(10)$ \\
\hline \multicolumn{2}{|l|}{ Radiographic features } \\
\hline Cavitation & $1(1)$ \\
\hline Atelectasis & $8(6)$ \\
\hline Pleural effusion & $27(20)$ \\
\hline Alveolar infiltrate pattern & $119(77)$ \\
\hline Interstitial infiltrate pattern & $1(1)$ \\
\hline Mixed infiltrate pattern & $3(2)$ \\
\hline Number of affected lobes, 1 & $83(61)$ \\
\hline Number of affected lobes, $\geq 2$ & $51(38)$ \\
\hline \multicolumn{2}{|l|}{ Vital signs, mean \pm SD } \\
\hline Respiratory rate & $29 \pm 7$ \\
\hline Heart rate & $96 \pm 19$ \\
\hline Systolic blood pressure, $\mathrm{mm} \mathrm{Hg}$ & $129 \pm 28$ \\
\hline Diastolic blood pressure, $\mathrm{mm} \mathrm{Hg}$ & $69 \pm 14$ \\
\hline \multicolumn{2}{|l|}{ Analytical data } \\
\hline Leucocytes $>12 \times 10^{9} / \mathrm{l}, \mathrm{n}(\%)$ & $76(56)$ \\
\hline Creatinine, mg/dl & $1.5 \pm 2.0$ \\
\hline C-reactive protein, $\mathrm{mg} / \mathrm{dl}$ & $20 \pm 13$ \\
\hline Platelets, $\times 10^{3} / 1$ & $277 \pm 111$ \\
\hline $\mathrm{Na}, \mathrm{mEq} / \mathrm{l}$ & $138 \pm 7$ \\
\hline $\mathrm{K}, \mathrm{mEq} / \mathrm{l}$ & $4.2 \pm 0.7$ \\
\hline Haematocrit, \% & $38 \pm 6$ \\
\hline Serum proteins, $g / l$ & $60 \pm 6$ \\
\hline Albumin, g/l & $33 \pm 7$ \\
\hline
\end{tabular}

Enterobacteriaceae (E coli and K pneumoniae) (9\%). L pneumophila, respiratory virus and MRSA were isolated in three cases each $(5 \%)$. Mixed aetiology was detected in four patients, including two cases of $S$ pneumoniae and a respiratory virus, one of $E$ coli and $C$ pneumoniae, and one of MRSA and $K$ pneumoniae. $H$ influenzae was isolated in only two patients (4\%).

An antibiotic resistance pattern was available in 32 patients, including 22 cases of $S$ pneumoniae, 3 MRSA, 1 P aeruginosa, $2 E$ coli, $1 \mathrm{~K}$ pneumoniae, $2 \mathrm{H}$ influenzae and 1 Providencia stuartii.

Microbial aetiology in deceased patients was determined in only 10 cases $(33 \%)$ and did not differ significantly from that of survivors, since $S$ pneumoniae was the most frequently isolated microorganism $(\mathrm{n}=5 ; 50 \%)$ followed by GNB (2 cases of $K$ pneumoniae, $1 E$ coli and $1 P$ stuartii) and MRSA $(\mathrm{n}=1)$.

Initial antibiotic treatment was aimed at CAP coverage, except for six patients who received a broad-spectrum antibiotic (ie, carbapenem), and included combined $\beta$-lactam + quinolone (30\%), $\beta$-lactam alone (25\%), quinolone alone $(17 \%)$ and combined $\beta$-lactam+macrolide (11\%). Accordingly, the most frequent causes of empiric antibiotic inadequacy were the use of $\beta$-lactams alone and lack of aspiration assessment in $25 \%$ and $15 \%$ of cases, respectively. 
Table 3 Severity indices

\begin{tabular}{|c|c|}
\hline & $\mathbf{n}(\%)^{*}$ \\
\hline \multicolumn{2}{|l|}{ Acute respiratory failure } \\
\hline $\mathrm{PaO}_{2} / \mathrm{Fi}_{2}<200$ & $16(16)$ \\
\hline Basal Sato ${ }_{2}<92 \%$ & $61(54)$ \\
\hline Acute renal failure (creatinine $>1.5 \mathrm{mg} / \mathrm{dl}$ ) & $28(21)$ \\
\hline Shock & $14(10)$ \\
\hline Multilobar infiltration & $51(38)$ \\
\hline 2 lobes affected & $42(31)$ \\
\hline$\geq 3$ lobes affected & $9(7)$ \\
\hline Admission to ICU & $13(9)$ \\
\hline Admission to intermediate care unit & $7(5)$ \\
\hline Fine score, $1-2$ & $9(6)$ \\
\hline Fine score, 3 & $12(8)$ \\
\hline Fine score, $4-5$ & $129(86)$ \\
\hline CRB-65 score, $0-1$ & $61(41)$ \\
\hline CRB-65 score, 2 & $61(41)$ \\
\hline CRB-65 score, 3-4 & 28 (19) \\
\hline Pneumonia severity index (PSI), mean \pm SD & $137 \pm 28$ \\
\hline
\end{tabular}

*Percentages of all cases with available information.

CRB-65, pneumonia severity score including: consciousness,

respiratory rate, blood pressure, 65 years of age cut-off; ICU, intensive care unit.

With regard to antibiotic resistance, we observed that initial antibiotic therapy was inappropriate in 12 patients (38\%); the antibiotic was changed on admission but there were no significant changes in mortality (only three patients with initial inadequate antibiotic therapy died during hospitalisation).

\section{Microbial aetiology}

Demographic, clinical and biochemical variables were compared for patients with typical CAP microbiology (ie, $S$ pneumoniae, $H$ influenzae, etc) $(\mathrm{n}=47)$ and those with potential first-line CAP antibiotic-resistant microorganisms (GNB or MRSA) $(n=11)$. No statistically significant differences were found between the two groups except for PSI score (132.6 \pm 25.3 for patients with typical CAP vs $157.7 \pm 30.0$ for patients with GNB or MRSA; t test; $\mathrm{p}=0.014$ ) and CRB-65, which was higher in patients with GNB or MRSA (classes 3-5: 13\% for patients with typical CAP vs $54 \%$ for patients with GNB or MRSA; $\left.\chi^{2} ; p=0.007\right)$. Statistically significant variables in the univariate analysis were PSI scoring (+1 point increase; OR 1.04; 95\% CI 1.01 to $1.07 ; \mathrm{p}=0.024)$,
CRB-65 classes 3-5 (OR 12.5; 95\% CI 2.0 to 78.0; $\mathrm{p}=0.007$ ) and length of stay ( +1 day increase; OR 1.08 ; $95 \%$ CI 0.99 to 1.17 ; $\mathrm{p}=0.075)$. No independent predictors of GNB or MRSA were found in the multivariate analysis.

\section{One-month mortality}

Demographic, clinical and biochemical variables were compared between patients who were deceased at 1 month $(n=30)$ and survivors. No significant differences were found between the two groups except for neurological co-morbidities (76\% for patients who had died at 1 month vs $49 \%$ for survivors; $\chi^{2}$; $\mathrm{p}=0.023$ ), and autonomy for daily activities (full: $6 \%$ for patients who died at 1 month vs $37 \%$ for survivors; $\chi^{2}$; $\mathrm{p}=0.023$ ). In the multivariate analysis (table 5), the independent predictors of 1 -month mortality were neurological diseases (OR 4.5 ; $95 \%$ CI 1.3 to $15.7 ; p=0.020$ ), septic shock (OR 6.6; 95\% CI 1.3 to $34.0 ; \mathrm{p}=0.025)$, pleural effusion (OR $3.6 ; 95 \%$ CI 1.1 to $11.7 ; \mathrm{p}=0.036)$ and isolation of GNB or MRSA (OR 16.4; $95 \% \mathrm{CI}$ 2.1 to $128.9 ; \mathrm{p}=0.008$ ) (table 5 ).

The $\chi^{2}$ goodness-of-fit analysis demonstrated the model's adequacy ( $p>0.05)$.

\section{DISCUSSION}

The peculiarities of $\mathrm{NH}$ populations and the increased risk of mortality have led NHAP to be considered a separate clinical entity, thereby justifying specific recommendations for clinical management ${ }^{25-27}$ and, recently, inclusion among HCAP. ${ }^{1}$ As in the literature, our population showed advanced age, numerous comorbidities (particularly neurological disorders), poor autonomy for daily activities (up to $70 \%$ of patients were partially or totally dependent) and, frequently, atypical clinical presentation (extrapulmonary manifestations). The role of age, comorbidities and functional status in this population thus appears to have a considerable effect on pneumonia severity and mortality. ${ }^{28}$ Many authors have shown that patients' functional status before admission is one of the most important prognostic factors for mortality ${ }^{6-32}$ and may considerably influence the decision of site of care ( $\mathrm{NH}$ vs hospital, ICU admission, etc) and aspects of clinical management (diagnostic procedures and lifeprolonging treatments). ${ }^{72633} 34$ We also observed that a history of neurological disorders (a major cause of the inability to perform daily activities) was an important prognostic factor of mortality. The highest mortality rate was recorded among

Table 4 Bacteriological findings of 57 nursing home patients (38\% of total population) with either probable or definitive aetiology of a pneumonia episode

\begin{tabular}{|c|c|c|c|c|c|c|c|c|}
\hline & n (\%) & Sputum & Blood & TBAS, BAL & Pleural fluid & Serology & Urinary antigen & N-P swab \\
\hline$S$ pneumoniae & $33(22)$ & 9 & 6 & 3 & 4 & & 19 & \\
\hline$H$ influenzae & $2(1)$ & 2 & 0 & 1 & 0 & & & \\
\hline MRSA & $3(2)$ & 2 & 1 & 1 & 0 & & & \\
\hline K pneumoniae & $2(1)$ & 0 & 1 & 2 & 0 & & & \\
\hline E coli & $3(2)$ & 0 & 1 & 2 & 1 & & & \\
\hline C pneumoniae & $1(1)$ & & & & & 2 & & \\
\hline L pneumophila & $3(2)$ & 0 & 0 & 0 & 0 & & 3 & \\
\hline Virus & $3(2)$ & & & & & 3 & & 2 \\
\hline P stuartii & $1(1)$ & 1 & & & & & & \\
\hline Candida albicans & $1(1)$ & 1 & 0 & 1 & 0 & & & \\
\hline Total & 57 (38) & 17 & 9 & 10 & 5 & 8 & 22 & 2 \\
\hline
\end{tabular}

Note: percentages refer to the total number of patients $(n=150)$.

BAL, bronchioalveolar lavage; MRSA, methicillin-resistant Staphylococcus aureus; N-P swab, naso-pharyngeal swab; TBAS, tracheobronchial aspirate. 
Table 5 Analysis of prognostic factors of 1-month mortality: significant univariate and multivariate associations

\begin{tabular}{|c|c|c|c|c|c|c|}
\hline & \multicolumn{3}{|c|}{ Univariate analysis } & \multicolumn{3}{|c|}{ Multivariate analysis } \\
\hline & $\overline{\mathbf{O R}}$ & $95 \% \mathrm{CI}$ & p Value & $\mathbf{O R}$ & $95 \% \mathrm{CI}$ & p Value \\
\hline Autonomy for daily activities & & & 0.078 & & & \\
\hline Full & 1.0 & - & - & & & \\
\hline Partial & 8.7 & 1.0 to 75.3 & 0.050 & & & \\
\hline None & 11.5 & 1.4 to 95.6 & 0.024 & & & \\
\hline Neurological diseases & 3.3 & 1.1 to 9.9 & 0.029 & 4.5 & 1.2 to 15.7 & 0.020 \\
\hline Septic shock & 3.0 & 0.9 to 10.1 & 0.078 & 6.6 & 1.2 to 34.0 & 0.025 \\
\hline Pleural effusion & 2.7 & 0.9 to 7.9 & 0.063 & 3.6 & 1.1 to 11.7 & 0.036 \\
\hline Pathogens & & & 0.036 & & & 0.028 \\
\hline Typical CAP & 1.0 & - & - & 1.0 & - & - \\
\hline $\mathrm{GNB}+\mathrm{MRSA}$ & 6.5 & 1.4 to 29.4 & 0.015 & 16.4 & 2.1 to 128.9 & 0.008 \\
\hline Unknown aetiology & 3.0 & 1.0 to 8.7 & 0.043 & 3.0 & 0.6 to 14.4 & 0.165 \\
\hline PSI scoring, +1 point & 1.02 & 1.01 to 1.05 & 0.029 & & & \\
\hline
\end{tabular}

patients admitted to the ward, suggesting that functional status, premorbid condition and, possibly, ethical considerations limited ICU admission of patients with poor life expectancy.

The mortality rate of our population (8\%) was very similar to ${ }^{14} 35$ or lower ${ }^{36}$ than that reported for CAP in the general population and in the elderly. In contrast, many studies on NHAP 1128 and the recent North American series on HCAP report higher mortality rates (up to $40 \%$ ). ${ }^{15} 16$ Interestingly, the Spanish study by Carratalà on HCAP, including $32 \mathrm{NH}$ patients (25.4\% of all HCAP), shows a mortality rate very close to that of this study $(10.3 \%$ for HCAP and $4 \%$ for CAP $) .{ }^{17}$ This similarity suggests two considerations: first, the considerable differences among these studies may denote a geographic variation of pathogens, virulence and antibiotic resistances. Secondly, no unique definition of $\mathrm{NH}$ is currently available worldwide and it may not be possible given the organisational differences in local healthcare systems and LTCFs in different countries. Therefore, both the geographic distribution of pathogens and differences in $\mathrm{NH}$ populations from different countries may partly explain such remarkable differences in mortality.

A central issue regarding NHAP and HCAP is microbial aetiology and, consequently, the empirical antibiotic treatment to use in clinical practice. Before guidelines for HAP, ventilatorassociated pneumonia (VAP) and HCAP, published in $2005,{ }^{1}$ recommendations for NHAP antibiotic treatment closely resembled CAP guidelines. ${ }^{23} 37$ Since the North American studies $^{15} 16$ reported a very high incidence of MDR infections among HCAP, an intense debate arose on the microbial aetiology of NHAP. Subsequently, American guidelines suggested empirically treating HCAP as a nosocomial infection. ${ }^{1}$

However, analysing the American retrospective studies on $\mathrm{HCAP}^{15} 16$ it is clear that the high rates of ICU admission, ventilatory support and mortality (pneumonia severity), the high incidence of GNB, even in patients with CAP, and the scarce information on microbiological methods make these data poorly comparable with ours.

The literature shows that, with the exception of two American studies on elderly patients with severe NHAP, ${ }^{10}{ }^{11}$ no other studies confirm the hypothesis of a nosocomial pattern in NHAP and HCAP.9 1117 Venditti et al ${ }^{38}$ show that receiving empirical antibiotic treatment not recommended by the latest HCAP guidelines was independently associated with increased mortality. Surprisingly, no microbiological data are shown in that study, suggesting that clinical conditions before hospitalisation, comorbidities or any other possible confounding factor may explain these results.

This is one of the largest NHAP series of the last decade and clearly shows that, in Spain, $S$ pneumoniae is still the most frequent organism causing pneumonia. Unfortunately, the rate of positive microbiological findings is fairly low, but similar to many other series of $\mathrm{NHAP}^{7}$ for different reasons, such as the following: (1) a poor cough reflex and an altered mental status considerably reduce availability of sputum samples in $\mathrm{NH}$ patients; (2) blood cultures are usually performed only in patients with fever, and elderly patients commonly have fewer temperature alterations in response to infection than younger individuals; and (3) pneumococcal and $L$ pneumophila urinary antigens are the most common source of aetiological diagnosis but were introduced only in late 2000. Some infrequent bacteria, such as $P$ aeruginosa and MRSA, can be easily detected even in poor-quality biological samples (ie, sputum), thereby increasing the number of cases with known aetiology.

It is also worth noting that empirical treatment was mainly concordant with CAP guidelines ${ }^{23}$ and, depending on antibiotic resistance, was inappropriate only in a few cases, with no increased mortality. Moreover, the microbial pattern did not differ between survivors and deceased patients, showing that the effect of aetiology on mortality in our series is probably slight.

However, it is important to underline that isolation of unusual microorganisms such as GNB or MRSA (potentially not susceptible to first-line CAP antibiotic treatment), which are more frequent in patients with higher severity scores, was associated with a considerable increase in the mortality risk.

Therefore, we still need to be cautious with therapeutic recommendations: a careful evaluation of possible risk factors for MDR infections in patients with NHAP, such as previous antibiotic treatment, recent hospitalisation or advanced chronic respiratory disease (ie, bronchiectasis or chronic obstructive pulmonary disease) and functional status before admission, appears essential to guide the use of broad-spectrum antibiotics on an individual basis. ${ }^{31}$ Moreover, functional status before admission should always be investigated in the process to decide diagnostic procedures and site of care. 
The following are possible limitations of this study:

1. The introduction of the urinary antigen detection method for $S$ pneumoniae and for $L$ pneumophila only in 2000 may have influenced the rate of aetiological findings in the first 3 years of this series.

2. The initial study design did not consider a case-control match with patients with CAP that might have complemented clinical and microbiological information on NHAP.

3. This is a single-centre Spanish study and our data may not be representative of Europe.

In conclusion, our $\mathrm{NH}$ patients appeared more similar to those affected by HAP in terms of age, functional status and comorbidities; however, low mortality was recorded. A 'community' microbial pattern was observed in NHAP, with the only exception of Enterobacteriaceae being slightly more frequent than in CAP. Additionally, isolation of GNB and MRSA was associated with a considerable increase in mortality risk.

These findings suggest that 'community-based' empirical antibiotic treatment is still indicated in NHAP, but additional risk factors for MDR infections (eg, previous antibiotic treatment and recent hospitalisation) and the risk of aspiration should always be assessed on an individual basis in order to guide the selection of broad-spectrum antibiotic treatment.

Further investigation with sound microbiological methodology is needed in NHAP to properly evaluate risk factors for MDR infections and optimise empirical antibiotic therapy.

Competing interests None.

Provenance and peer review Not commissioned; externally peer reviewed.

\section{REFERENCES}

1. American Thoracic Society. Guidelines for the management of adults with hospital-acquired, ventilator-associated, and healthcare-associated pneumonia. Am J Respir Crit Care Med 2005;171:388-416.

2. Richards C. Infections in residents of long-term care facilities: an agenda for research. Report of an expert panel. J Am Geriatr Soc 2002;50:570-6.

3. Gross JS, Neufeld RR, Libow LS, et al. Autopsy study of the elderly institutionalized patient. Review of 234 autopsies. Arch Intern Med 1988;148:173-6.

4. Kerr HD, Byrd JC. Nursing home patients transferred by ambulance to a VA emergency department. J Am Geriatr Soc 1991;39:132-6.

5. Marrie TJ, Durant $H$, Yates L. Community-acquired pneumonia requiring hospitalization: 5-year prospective study. Rev Infect Dis 1989;11:586-99.

6. Mylotte JM. Nursing home-acquired pneumonia. Clin Infect Dis 2002;35: 1205-11.

7. Muder RR. Pneumonia in residents of long-term care facilities: epidemiology, etiology, management, and prevention. Am J Med 1998;105:319-30.

8. Stevenson KB. Regional data set of infection rates for long-term care facilities: description of a valuable benchmarking tool. Am J Infect Control 1999;27 20-6.

9. Lim WS, Macfarlane JT. A prospective comparison of nursing home acquired pneumonia with community acquired pneumonia. Eur Respir J 2001;18 362-8.

10. El Solh AA, Sikka P, Ramadan F, et al. Etiology of severe pneumonia in the very elderly. Am J Respir Crit Care Med 2001;163:645-51.

11. Martinez-Moragon E, Garcia FL, Serra SB, et al. [Community-acquired pneumonia among the elderly: differences between patients living at home and in nursing homes]. Arch Bronconeumol 2004:40:547-52.

12. Niederman MS, Brito V. Pneumonia in the older patient. Clin Chest Med 2007:28:751-71, vi.

13. Donowitz GR, Cox HL. Bacterial community-acquired pneumonia in older patients. Clin Geriatr Med 2007;23:515-34, vi.
14. Fernandez-Sabe N, Carratala J, Roson B, et al. Community-acquired pneumonia in very elderly patients: causative organisms, clinical characteristics, and outcomes. Medicine (Baltimore) 2003;82:159-69.

15. Kollef MH, Shorr A, Tabak YP, et al. Epidemiology and outcomes of health-careassociated pneumonia: results from a large US database of culture-positive pneumonia. Chest 2005:128:3854-62.

16. Micek ST, Kollef KE, Reichley RM, et al. Health care-associated pneumonia and community-acquired pneumonia: a single-center experience. Antimicrob Agents Chemother 2007:51:3568-73.

17. Carratala J, Mykietiuk A, Fernandez-Sabe N, et al. Health care-associated pneumonia requiring hospital admission: epidemiology, antibiotic therapy, and clinical outcomes. Arch Intern Med 2007:167:1393-9.

18. Marrie TJ. Pneumonia in the long-term-care facility. Infect Control Hosp Epidemio 2002:23:159-64

19. Irvine PW, Van BN, Crossley K. Causes for hospitalization of nursing home residents: the role of infection. J Am Geriatr Soc 1984;32:103-7.

20. El-Solh AA, Aquilina AT, Dhillon RS, et al. Impact of invasive strategy on management of antimicrobial treatment failure in institutionalized older people with severe pneumonia. Am J Respir Crit Care Med 2002;166:1038-43.

21. Tambyah PA, Habib AG, Ng TM, et al. Community-acquired methicillin-resistant Staphylococcus aureus infection in Singapore is usually 'healthcare associated'. Infect Control Hosp Epidemiol 2003;24:436-8.

22. Naimi TS, LeDell KH, Como-Sabetti K, et al. Comparison of community- and health care-associated methicillin-resistant Staphylococcus aureus infection. JAMA 2003;290:2976-84.

23. Mandell LA, Wunderink RG, Anzueto A, et al. Infectious Diseases Society of America/American Thoracic Society consensus guidelines on the management of community-acquired pneumonia in adults. Clin Infect Dis 2007:44 (Suppl 2):S27-72.

24. Rano A, Agusti C, Jimenez $P$, et al. Pulmonary infiltrates in non-HIV immunocompromised patients: a diagnostic approach using non-invasive and bronchoscopic procedures. Thorax 2001;56:379-87.

25. Niederman MS, Mandell LA, Anzueto A, et al. Guidelines for the management of adults with community-acquired pneumonia. Diagnosis, assessment of severity, antimicrobial therapy, and prevention. Am J Respir Crit Care Med 2001;163:1730-54.

26. Muder RR, Aghababian RV, Loeb MB, et al. Nursing home-acquired pneumonia: an emergency department treatment algorithm. Curr Med Res Opin 2004:20:1309-20.

27. Furman CD, Rayner AV, Tobin EP. Pneumonia in older residents of long-term care facilities. Am Fam Physician 2004;70:1495-500

28. Muder RR, Brennen C, Swenson DL, et al. Pneumonia in a long-term care facility. A prospective study of outcome. Arch Intern Med 1996;156:2365-70.

29. Marrie TJ, Blanchard W. A comparison of nursing home-acquired pneumonia patients with patients with community-acquired pneumonia and nursing home patients without pneumonia. J Am Geriatr Soc 1997:45:50-5.

30. Naughton BJ, Mylotte JM, Tayara A. Outcome of nursing home-acquired pneumonia: derivation and application of a practical model to predict 30 day mortality. J Am Geriatr Soc 2000;48:1292-9.

31. Brito V Niederman MS. Healthcare-associated pneumonia is a heterogeneous disease, and all patients do not need the same broad-spectrum antibiotic therapy as complex nosocomial pneumonia. Curr Opin Infect Dis 2009;22:316-25.

32. El Solh AA, Pietrantoni $\mathrm{C}$, Bhat $\mathrm{A}$, et al. Indicators of potentially drug-resistant bacteria in severe nursing home-acquired pneumonia. Clin Infect Dis 2004:39:474-80

33. Dosa D. Should I hospitalize my resident with nursing home-acquired pneumonia? J Am Med Dir Assoc 2006;7(3 Suppl):S74-80, 73.

34. van der Steen JT, Ooms ME, Ader HJ, et al. Withholding antibiotic treatment in pneumonia patients with dementia: a quantitative observational study. Arch Intern Med 2002;162:1753-60.

35. Ruiz M, Ewig S, Marcos MA, et al. Etiology of community-acquired pneumonia: impact of age, comorbidity, and severity. Am J Respir Crit Care Med 1999:160:397-405.

36. Ewig S, Birkner N, Strauss R, et al. New perspectives on community-acquired pneumonia in 388,406 patients. Thorax 2009;64:1062-9.

37. Naughton BJ, Mylotte JM. Treatment guideline for nursing home-acquired pneumonia based on community practice. J Am Geriatr Soc 2000;48:82-8.

38. Venditti M, Falcone M, Corrao S, et al. Outcomes of patients hospitalized with community-acquired, health care-associated, and hospital-acquired pneumonia. Ann Intern Med 2009;150:19-26. 


\section{REFERENCES}

1. Anderson SD. Exercise-induced asthma. In: Kay AB, ed. Allergy and allergic diseases. Oxford: Blackwell Scientific Publications, 1997:672-711.

2. Rundell KW, Im J, Mayers LB, et al. Self-reported symptoms and exercise-induced asthma in the elite athlete. Med Sci Sports Exerc 2001; 33:208-13.

3. Dickinson JW, Whyte GP, McConnell AK, et al. Midexpiratory flow versus FEV1 measurements in the diagnosis of exercise induced asthma in elite athletes. Thorax 2006;61:111-4.

4. Holzer K, Douglass JA. Exercise induced bronchoconstriction in elite athletes: measuring the fall. Thorax 2006;61:94-6.

5. Parsons JP, Mastronarde JG. Exercise-induced bronchoconstriction in athletes. Chest 2005;128:3966-74.

\section{Corrections}

doi:10.1136/thx.2009.122291 corr1

Conway Morris A, Kefala K, Wilkinson $\mathrm{TS}$, et al. Diagnostic importance of pulmonary interleukin-1b and interleukin-8 in ventilator-associated pneumonia. Thorax 2010;65:201-7. This article should have included the note that Dr Kefala was joint first author.
Polverino E, Dambrava P, Cilloniz C, et al. Nursing home-acquired pneumonia: a 10 year single-centre experience. Thorax 2010;65:354-59. The correct affiliation for affiliation 1 should have read "Respiratory Department, Hospital Clinic-IDIBAPS, Barcelona-Spain, Centro de Investigación Biomedica En RedEnfermedades Respiratorias (CibeRes, CB06/06/0028, el Ciberes es una iniciativa del ISCIII) - 2009SGRO http://www.idibapsrespiratoryresearch. org."

doi:10.1136/thx.2009.133108corr1

Millett C, Glantz SA. Assigning an '18' rating to movies with tobacco imagery is essential to reduce youth smoking. Thorax 2010;65:377-8. The authors referred to a paper by McNeil et al; this should have been Lyons et al (Lyons A, McNeill A, Chen Y, et al).
Lyons A, McNeill A, Chen Y, et al. Tobacco and tobacco branding in films most popular in the UK from 1989 to 2008. Thorax 2010;65:417-22. There is an error in figure legend 2 which currently reads "Trends in all tobacco intervals and tobacco use intervals per hour per day by British Board of Film Classification (BBFC) category (all figures expressed as means)." It should have read: "Trends in all tobacco intervals and tobacco use intervals per hour per year by British Board of Film Classification (BBFC) category (all figures expressed as means)."

doi:10.1136/thx.2009.127274corr1

Kemp SV, El Batrawy SH, Harrison RN, et al. Learning curves for endobronchial ultrasound using cusum analysis. Thorax 2010;65:534-8. The author name A Roselli should have read A Rosell. 